\title{
Laminados biodegradáveis de blendas de amido de mandioca e poli(vinil álcool): efeito da formulação sobre a cor e opacidade
}

\section{Sheets of cassava starch - poly (vinyl alcohol) produced by extrusion: effect of formulation on the color and opacity}

\author{
Juliano Zanela1*, Mônica Oliveira Reis', Adriana Passos Dias', Suzana Mali', \\ Maria Victória Eiras Grossmann ${ }^{1}$ e Fabio Yamashita ${ }^{1}$ \\ ${ }^{1}$ Departamento de Ciência e Tecnologia de Alimentos, Universidade Estadual de Londrina - UEL, \\ Londrina, PR, Brasil \\ *julianozanela@gmail.com
}

\section{Resumo}

Foram produzidos 12 laminados com diferentes proporções de amido de mandioca, poli(vinil álcool) (PVA) e glicerol por extrusão usando um planejamento de mistura. A opacidade dos materiais variou entre 31 e $56 \%$, e a concentração de PVA e a interação entre as concentrações de amido e PVA foram os principais responsáveis pelo aumento da opacidade. A diferença de cor $\left(\Delta \mathrm{E}^{*}\right)$ variou entre 20-30, sendo a concentração de amido o principal responsável pelo aumento da diferença de cor, por promover uma coloração mais amarelada nos laminados.

Palavras-chave: extrusão, biopolimeros, propriedades ópticas.

\section{Abstract}

Twelve sheets were produced by extrusion with different proportions of cassava starch, poly (vinyl alcohol) (PVA) and glycerol using a mixture design. The opacity of the materials ranged from 31 to $56 \%$, and the concentration of PVA and the interaction between starch and PVA concentrations the mainly responsible for increasing opacity. The color difference $\left(\Delta \mathrm{E}^{*}\right)$ ranged from 20 to 30 , the concentration of starch being primarily responsible for increasing in color difference by promoting a more yellowish color in sheets.

Keywords: extrusion, biopolymers, optical properties.

\section{Introdução}

Há uma crescente busca pelo desenvolvimento de polímeros biodegradáveis para a substituição dos polímeros convencionais devido aos problemas ambientais e escassez de petróleo. O amido é um biopolímero bastante estudado no desenvolvimento de materiais biodegradáveis, devido ao seu baixo custo e abundância. $\mathrm{O}$ amido não é um termoplástico verdadeiro, mas na presença de agentes plastificantes, calor e cisalhamento ele perde sua estrutura semicristalina, originando o amido termoplástico (ATp), que é uma material amorfo, com características semelhantes as observadas em polímeros sintéticos ${ }^{[1,2]}$.

Materiais produzidos somente com ATp apresentam propriedades mecânicas e barreira ao vapor de água inadequadas para produção e aplicação em escala comercial ${ }^{[3]}$. Dessa forma, é necessário produzir blendas com outros polímeros biodegradáveis para melhorar as propriedades do material, como o poli(vinil álcool) (PVA). Dependendo do grau de hidrólisee viscosidade do PVA, é possível obter materiais com diferentes propriedades ${ }^{[4]}$.
Uma característica importante dos materiais biodegradáveis é a opacidade, principalmente para aqueles que vão ser utilizados como embalagem, pois a transparência é importante para aqueles produtos que precisam ser visualizados pelo consumidor. Já para os produtos sensíveis à fotodegradação, é importante que a embalagem seja opaca ${ }^{[5]}$.

O objetivo foi estudar a influência das concentrações de amido, glicerol e PVA sobre a cor e opacidade de laminados produzidos por extrusão.

\section{Experimental}

Foi utilizado amido de mandioca (Indemil, Brasil), glicerol (Dinâmica, Brasil) e poli(vinil álcool) Selvol 203 (Sekisui Chemical, Japão), com grau de hidrólise de 88,14\% e viscosidade de $4,10 \mathrm{cP}$ em solução a $4 \%$, e as formulações estão na Tabela 1. As misturas foram homogeneizadas manualmente e mantidas em estufa à vácuo (Quimis, Brasil), por uma hora a $85^{\circ} \mathrm{C}$ para melhorar a incorporação do 
glicerol, segundo metodologia proposta por Jang e Lee ${ }^{[6]}$. Após esta etapa, as blendas foram processadas em extrusora dupla-rosca co-rotativa modelo D-20 (BGM, Brasil), com velocidade dos parafusos de $100 \mathrm{rpm}$ e perfil de temperatura de $90 / 170 / 170 / 170 / 170^{\circ} \mathrm{C}$, equipada com uma matriz do tipo "flat die" com abertura de $0,8 \mathrm{~mm}$, para a produção de laminado em conjunto com uma calandra de três rolos (AX Plásticos, Brasil). As velocidades dos rolos tracionadores da calandra foram definidas individualmente para cada tratamento, para garantir um laminado contínuo, coeso e de espessura homogênea.

As análises de cor e opacidade foram realizadas conforme metodologia descrita por Sobral ${ }^{[7]}$, oito replicatas de cada laminado foram analisadas utilizando um colorímetro BYK Gardner (Alemanha), com um ângulo de $10^{\circ}$ e iluminante D65.

A opacidade (Y) foi determinada como a relação entre as leituras de opacidade das amostras sobre os padrões preto $\left(\mathrm{Y}_{\mathrm{p}}\right)$ e branco $\left(\mathrm{Y}_{\mathrm{b}}\right)$, sendo os resultados expressos em escala arbitrária (0-100\%) conforme Equação 1:

$$
Y=\left(Y_{p} / Y_{b}\right) * 100
$$

A análise de cor foi determinada como a diferença de cor $\left(\Delta \mathrm{E}^{*}\right)$, os parâmetros $\Delta \mathrm{L}^{*}, \Delta \mathrm{a}^{*} \mathrm{e} \Delta \mathrm{b}^{*}$ foram determinados pela Equação 2, como a diferença entre as leituras do padrão branco e das amostras sobre esse padrão.

$$
\Delta \mathrm{E}^{*}=\left[(\Delta \mathrm{L} *)^{2}+(\Delta \mathrm{a} *)^{2}+(\Delta \mathrm{b} *)^{2}\right]^{0,5}
$$

$\mathrm{O}$ ajuste do modelo do planejamento de mistura foi realizado utilizando o software Statistica 7.0 (Statsoft, EUA).

\section{Resultados e Discussão}

A opacidade variou de 31 a 56\%, já a diferença de cor variou entre 20 e 30 quando comparado ao padrão de cor branca (Tabela 1).

A Figura 1 apresenta as superfícies de resposta e os coeficientes do modelo, sendo possível observar que o PVA foi o componente puro de maior efeito para o modelo $(38,86)$,

\begin{tabular}{|c|c|c|c|c|c|c|c|c|}
\hline \multirow{2}{*}{ Código } & \multicolumn{3}{|c|}{ Componente real } & \multicolumn{3}{|c|}{ Pseudo-componente } & \multicolumn{2}{|c|}{ Resposta } \\
\hline & Amido & PVA & Glicerol & Amido & PVA & Glicerol & Opacidade & $\Delta \mathbf{E}^{*}$ \\
\hline T 1 & 0,675 & 0,075 & 0,250 & 0,944 & 0,056 & 0,000 & $39( \pm 2)$ & $30( \pm 3)$ \\
\hline T 2 & 0,630 & 0,070 & 0,300 & 0,778 & 0,037 & 0,185 & $34( \pm 4)$ & $27( \pm 2)$ \\
\hline T 3 & 0,585 & 0,065 & 0,350 & 0,611 & 0,018 & 0,370 & $36( \pm 4)$ & $27( \pm 4)$ \\
\hline T 4 & 0,540 & 0,060 & 0,400 & 0,444 & 0,000 & 0,556 & $31( \pm 6)$ & $22( \pm 3)$ \\
\hline T 5 & 0,600 & 0,150 & 0,250 & 0,667 & 0,333 & 0,000 & $55( \pm 4)$ & $28( \pm 5)$ \\
\hline T 6 & 0,560 & 0,140 & 0,300 & 0,518 & 0,296 & 0,185 & $48( \pm 2)$ & $28( \pm 2)$ \\
\hline T 7 & 0,520 & 0,130 & 0,350 & 0,370 & 0,259 & 0,370 & $43( \pm 3)$ & $22( \pm 2)$ \\
\hline T 8 & 0,480 & 0,120 & 0,400 & 0,222 & 0,222 & 0,556 & $41( \pm 2)$ & $23( \pm 2)$ \\
\hline T 9 & 0,525 & 0,225 & 0,250 & 0,389 & 0,611 & 0,000 & $56( \pm 3)$ & $25( \pm 3)$ \\
\hline T 10 & 0,490 & 0,210 & 0,300 & 0,259 & 0,556 & 0,185 & $49( \pm 2)$ & $22( \pm 3)$ \\
\hline T 11 & 0,455 & 0,195 & 0,350 & 0,130 & 0,500 & 0,370 & $46( \pm 3)$ & $21( \pm 2)$ \\
\hline T 12 & 0,420 & 0,180 & 0,400 & 0,000 & 0,444 & 0,556 & $44( \pm 4)$ & $20( \pm 2)$ \\
\hline
\end{tabular}

Tabela 1. Componentes reais, pseudo-componentes e respostas do planejamento de mistura.

\section{Opacidade (\%)}

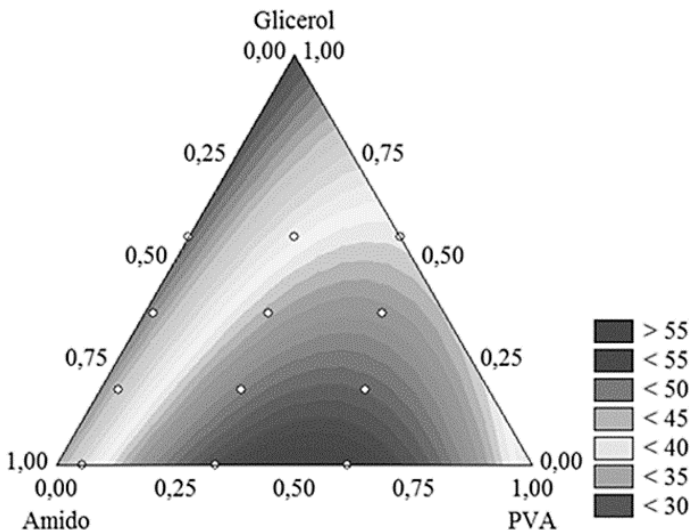

Opacidade $(\%)=34,39 \beta_{1}+38,86 \beta_{2}+28,87 \beta_{3}$ $+78,03 \beta_{12}+37,30 \beta_{13} \quad R^{2}=0,82$

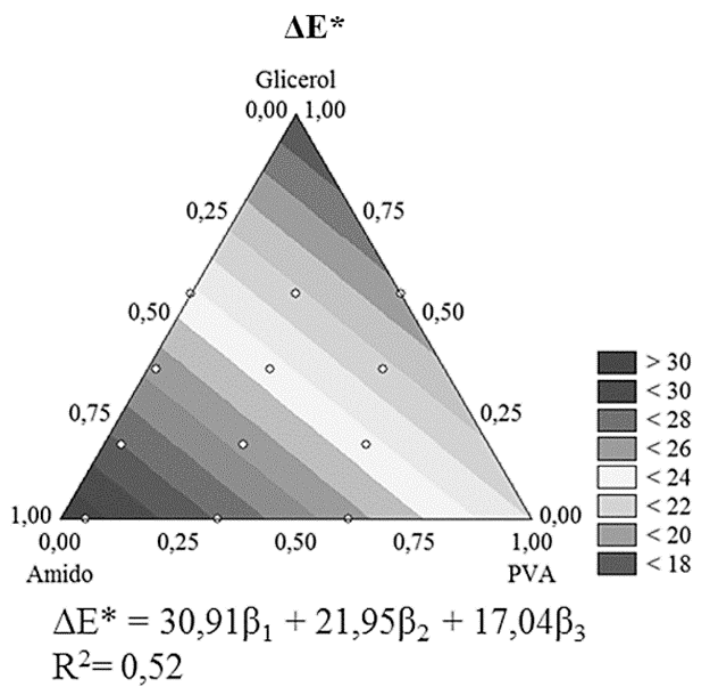

Figura 1. Superfície de resposta das variáveis opacidade e diferença de cor em função da concentração de amido, PVA e glicerol, em termos de seus pseudo-componentes. $\beta_{1}=$ amido, $\beta_{2}=$ PVA, $\beta_{3}=$ glicerol, $\beta_{12}=$ interação amido + PVA, $\beta_{13}=$ interação amido + glicerol. 
bem como a interação amido/PVA $(78,03)$, apresentando ainda um bom ajuste aos dados experimentais $\left(\mathrm{R}^{2}=0,82\right)$. Para a variável $\Delta \mathrm{E}^{*}$, o amido $(30,91)$ apresentou o maior efeito, pois laminados com maiores proporções de amido apresentaram coloração mais amarelada, enquanto que laminados com maiores concentrações de PVA apresentaram coloração mais esbranquiçada, resultando em menor $\Delta \mathrm{E}^{*}$ e maior opacidade.

Moraes et al. ${ }^{[8]} \mathrm{e}$ Silva et al. ${ }^{[9]} \mathrm{em}$ filmes de PVA e gelatina plastificados com glicerol e obtidos por casting obtiveram valores máximos de opacidade de 2,2 e $2,4 \%$ respectivamente, e $\Delta \mathrm{E}^{*}$ de no máximo 3,0 e 3,6 , respectivamente, muito inferiores aos obtidos no presente estudo, isso se deve ao uso de gelatina ao invés de amido, a menor espessura dos materiais obtidos, bem como a obtenção pela metodologia de casting, que promove a formação de materiais mais uniformes, transparentes e menos espessos quando comparados aos laminados obtidos por extrusão. Devido a sua estrutura, a gelatina ao contrário do amido não apresenta cristalinidade, permitindo uma maior passagem da luz por estar em um estado totalmente amorfo ${ }^{[10]}$, porém tanto o PVA quando o amido sofrem recristalização, isso reduz a passagem de luz, aumentando consequentemente sua opacidade.

O amido produz filmes mais opacos conforme observado por Fakhoury et al. ${ }^{[10]}$, quando comparou diferentes proporções de amido e gelatina em filmes obtidos por casting e plastificados com glicerol, em que com o aumento do percentual de amido em relação a gelatina foi observado um aumento da opacidade, de aproximadamente 7 a $24 \%$ com a relação amido/gelatina passando de $1 / 4$ para $4 / 1$ respectivamente. O mesmo comportamento foi observado por Garcia et al. ${ }^{[1]}$ em filmes de amido e quitosana, onde o amido promoveu um aumento da opacidade dos materiais.

Rocha et al ${ }^{\left[{ }^{12]}\right.}$ produziram filmes de amido de mandioca e proteína de soja plastificados com glicerol pela técnica de casting e observaram que com o aumento da concentração de proteína de soja houve aumento da opacidade aparente e do $\Delta \mathrm{E}^{*}$ (variando de 8,14 a 17,62) dos filmes, e conforme os autores essa diferença foi devido à coloração amarelada característica da proteína de soja. Almeida et al. ${ }^{[13]}$ produziram filmes de amido de batata e celulose microbiana plastificados com glicerol pela técnica de casting e observaram uma maior opacidade nos filmes em função do aumento da concentração de celulose microbiana nos filmes. Ambos os autores supracitados observaram que o amido não foi um fator importante para a opacidade e o $\Delta \mathrm{E}^{*}$ dos filmes, comportamento contrário ao observado no presente trabalho e essa diferença pode ser atribuída ao processo de produção dos materiais. A técnica de casting tende a produzir materiais mais translúcidos de que aqueles obtidos por extrusão, onde podem ocorrer reações de degradação do amido ou do PVA devido às altas temperaturas e taxas de cisalhamento, tornando os materiais com coloração mais escura e mais opaca de que filmes produzidos por casting.

A espessura também é um fator determinante para o aumento da opacidade, Mali et al..$^{[14]}$, observaram um aumento da opacidade em filmes de amido de cará plastificados com glicerol com o aumento da espessura dos mesmos, sendo os materiais obtidos no presente estudo apresentaram espessura média de $858 \mu \mathrm{m}$, promovendo assim um aumento esperado na opacidade e diferença de cor.

\section{Conclusões}

Os laminados biodegradáveis a base de amido e PVA obtidos por extrusão se mostraram levemente opacos, sendo o PVA e a interação amido/PVA os principais responsáveis pelo aumento da opacidade dos laminados. A diferença de cor é devida principalmente ao efeito do amido, que promoveu materiais de coloração mais amarelada.

\section{Agradecimentos}

Os autores agradecem a Universidade Tecnológica Federal do Paraná, ao CNPq, CAPES e Fundação Araucária pelas bolsas de estudo e apoio financeiro.

\section{Referências}

1. Liu, H., Xie, F., Yu, L., Chen, L., \& Li, L. (2009). Thermal processing of starch-based Polymers. Progress in Polymer Science, 34(12), 1348-1368. http://dx.doi.org/10.1016/j. progpolymsci.2009.07.001.

2. Olivato, J. B., Müller, C. M. O., Yamashita, F., Grossmann, M. V. E., \& Nobrega, M. M. (2013). Study of the compatibilizer effect in the properties of starch/polyester blends. Polimeros: Ciência e Tecnologia, 23(3), 346-351. http://dx.doi.org/10.4322/ polimeros.2013.014

3. Brandelero, R. P. H., Yamashita, F., \& Grossmann, M. V. E. (2010). Effect of the method of production of the blends on mechanical and structural properties of biodegradable starch films produced by blown extrusion. Carbohydrate Polymers, 82(4), 1102-1109. http://dx.doi.org/10.1016/j.carbpol.2010.06.034.

4. Maria, T. M. C., Carvalho, R. A., Sobral, P. J. A., Habitante, A. M. B. Q., \& Solorza-Faria, J. (2008). The effect of the degree of hydrolysis of the PVA and the plasticizer concentration on the color, opacity, and thermal and mechanical properties of films based on PVA and gelatin blends. Journal of Food Engineering, 87(2), 191-199. http://dx.doi.org/10.1016/j. jfoodeng.2007.11.026.

5. Pelissari, F. M., Yamashita, F., Garcia, M. A., Martino, M. N., Zaritzky, N. E., \& Grossmann, M. V. E. (2012). Constrained mixture design applied to the development of cassava starch - chitosan blown films. Journal of Food Engineering, 108(2), 262-267. http://dx.doi.org/10.1016/j.jfoodeng.2011.09.004.

6. Jang, J., \& Lee, D. (2003). Plasticizer effect on the melting and crystallization behavior of polyvinyl alcohol. Polymer, 44(26), 8139-8156. http://dx.doi.org/10.1016/j.polymer.2003.10.015.

7. Sobral, P. J. A. (2000). Influência da espessura de biofilmes feitos à base de proteínas miofibrilares sobre suas propriedades funcionais. Pesquisa Agropecuaria Brasileira, 35(6), 12511259. http://dx.doi.org/10.1590/S0100-204X2000000600022.

8. Moraes, I., Silva, G. G. D. I., Carvalho, R. A., Habitante, A. N. Q. B., Bergo, P. V. A., \& Sobral, P. V. A. (2008). Influência do grau de hidrólise do poli(vinil álcool) nas propriedades físicas de filmes à base de blendas de gelatina e poli(vinil álcool) plastificados com glicerol. Ciência e Tecnologia de Alimentos, 28(3), 738-745. http://dx.doi.org/10.1590/S010120612008000300034.

9. Silva, G. G. D. I., Sobral, P. J. A., Carvalho, R. A., Bergo, P. V. A., Mendieta-Taboada, O., \& Habitante, A. M. Q. B. (2008). Biodegradable films based on blends of gelatin and poly (vinyl alcohol): effect of PVA type or concentration on some physical properties of films. Journal of Polymers and the Environment, 16(4), 276-285. http://dx.doi.org/10.1007/ s10924-008-0112-9. 
10. Fakhoury, F. M., Martelli, S. M., Bertan, L. C., Yamashita, F., Mei, L. H. I., \& Queiroz, F. P. C. (2012). Edible films made from blends of manioc starch and gelatin - Influence of different types of plasticizer and different levels of macromolecules on their properties. $L W T-$ Food Science and Technology, 49(1), 149-154. http://dx.doi.org/10.1016/j.lwt.2012.04.017.

11. Garcia, M. A., Pinotti, A., \& Zaritzky, N. (2006). Physicochemical, water vapor barrier and mechanical properties of corn starch and chitosan composite films. Starch, 58(9), 453-463. http:// dx.doi.org/10.1002/star.200500484.

12. Rocha, G. O., Farias, M. G., Carvalho, C. W. P., Ascheri, J. L. R., \& Galdeano, M. C. (2014). Filmes compostos biodegradáveis a base de amido de mandioca e proteína de soja. Polímeros: Ciência e Tecnologia, 24(5), 587-595. http:// dx.doi.org/10.1590/0104-1428.1355.
13. Almeida, D. M., Woiciechowski, A. L., Wosiacki, G., Prestes, R. A., \& Pinheiro, L. A. (2013). Propriedades físicas, químicas e de barreira em filmes formados por blenda de celulose bacteriana e fécula de batata. Polímeros: Ciência e Tecnologia, 23(4), 538-546. http://dx.doi.org/10.4322/polimeros.2013.038.

14. Mali, S., Grossmann, M. V. E., Garcia, M. A., Martino, M. N., \& Zaritzky, N. E. (2004). Barrier, mechanical and optical properties of plasticized yam starch films. Carbohydrate Polymers, 56(2), 129-135. http://dx.doi.org/10.1016/j.carbpol.2004.01.004.

Enviado: Jun. 25, 2014

Revisado: Jan. 12, 2015

Aceito: Fev. 04, 2015 